\title{
Total laparoscopic hysterectomy of very enlarged uterus (3030 g): case report and review of the literature
}

\author{
Marcello Ceccaroni ${ }^{1}$, Giovanni Roviglione $^{1}$, Anna Pesci $^{2}$, Sara Quintana $^{3}$, Francesco Bruni ${ }^{1}$, Roberto Clarizia ${ }^{1}$ \\ ${ }^{1}$ Department of Obstetrics and Gynecology, Gynecologic Oncology and Minimally Invasive Pelvic Surgery Unit, \\ International School of Surgical Anatomy, “Sacred Heart” Hospital - Negrar, Verona, Italy \\ 2Department of Human Pathology, "Sacred Heart" Hospital - Negrar, Verona, Italy \\ ${ }^{3}$ Department of Obstetrics and Gynecology, Hospital Ferrol, La Coruña, Italy
}

Videosurgery Miniinv 2014; 9 (2): 302-307

DOI: $10.5114 /$ wiitm.2014.43026

\begin{abstract}
Fibromatosis is the most frequent benign uterine pathology of fertile women, rarely causing anomalous enlargement of the uterus. Traditionally the surgical treatment has been abdominal hysterectomy. However, development of minimally invasive techniques has led to major safeness of the laparoscopic route. We report a case of total laparoscopic hysterectomy performed on a uterus weighting more than 3,000 $\mathrm{g}$ and present a review of the literature about the laparoscopic approach to very enlarged uteri.
\end{abstract}

Key words: enlarged uterus, total laparoscopic, hysterectomy, laparoscopy, weight, morcellation, fibromatosis.

\section{Introduction}

Fibromatosis is the most frequent benign uterine pathology affecting fertile women, representing the most frequent indication for hysterectomy [1]. Treatment of fibroids is usually conservative with a minimally invasive approach through laparoscopy. However, abdominal hysterectomy is usually preferred in the case of very large uteri. Nowadays, improvements in minimally invasive techniques have increased the number of these patients who undergo laparoscopic hysterectomy [2].

Few reports exist in the literature on laparoscopic hysterectomy on a very large uterus. This report underlines the possibility of a laparoscopic approach for hysterectomy even in extremely large uteri.

\section{Case report}

A 48-year-old nulliparous woman was referred for stypsis, dyspepsia and metrorrhagia with a previous diagnosis of uterine myomas $(8 \mathrm{~cm}) 7$ years before. Personal history was negative for abdominal surgery and other health problems apart from obesity (body mass index (BMI) $33.8 \mathrm{~kg} / \mathrm{m}^{2}$ ). The patient was routinely followed by her general gynaecologist for an cervical dysplasia found at pap-smear and confirmed by colposcopic evaluation 3 years previously. The gynaecological examination showed a uterus with irregular shape and the fundus reached the right hypochondrium. The uterus was difficult to mobilize and moderately painful in its fundic part, where it presented an intramural/subserous myoma of $14 \mathrm{~cm}$. Combined transvaginal/transabdominal ultrasonography was performed showing multiple fibroids and normal adnexa. Blood tests excluded anaemia (haemoglobin was $13.7 \mathrm{~g} / \mathrm{dl}$ ).

Considering the presence of myomas and abnormal pap-smear, the patient was proposed a total laparoscopic hysterectomy with preservation of the adnexa.

\section{Address for correspondence:}

Marcello Ceccaroni MD, PhD, Department of Obstetrics and Gynecology, Gynecologic Oncology and Minimally Invasive Pelvic Surgery

Unit, International School of Surgical Anatomy, “Sacred Heart” Hospital - Negrar, Via Don A. Sempreboni 5, 37124 Negrar, Italy,

phone: +393479797201, fax: 0456014000, e-mail: issaschool@gmail.com 
The surgical procedure was performed in a lithotomic position, under general anaesthesia. A Minelli uterine manipulator (Karl Storz GmbH \& Co, Tuttlingen, Germany) was inserted transvaginally before beginning the operation and removed after colpotomy.

Initially, an umbilical trocar was placed after creation of pneumoperitoneum with a Veress needle to visualize the uterus. Therefore, we positioned a $10 \mathrm{~mm}$ trocar through a right subcostal access; thus a 30-degree optic was introduced to optimize the visualization and subsequently three additional $5 \mathrm{~mm}$ trocars were inserted, namely in the suprapubic region and bilaterally in the iliac fossae.

A huge uterus, reaching the right anterior border of the liver and deformed by multiple large intramural and subserous myomas, was visualized (Photo $1 \mathrm{~A}$ ). The adnexa were bilaterally normal and no free fluid
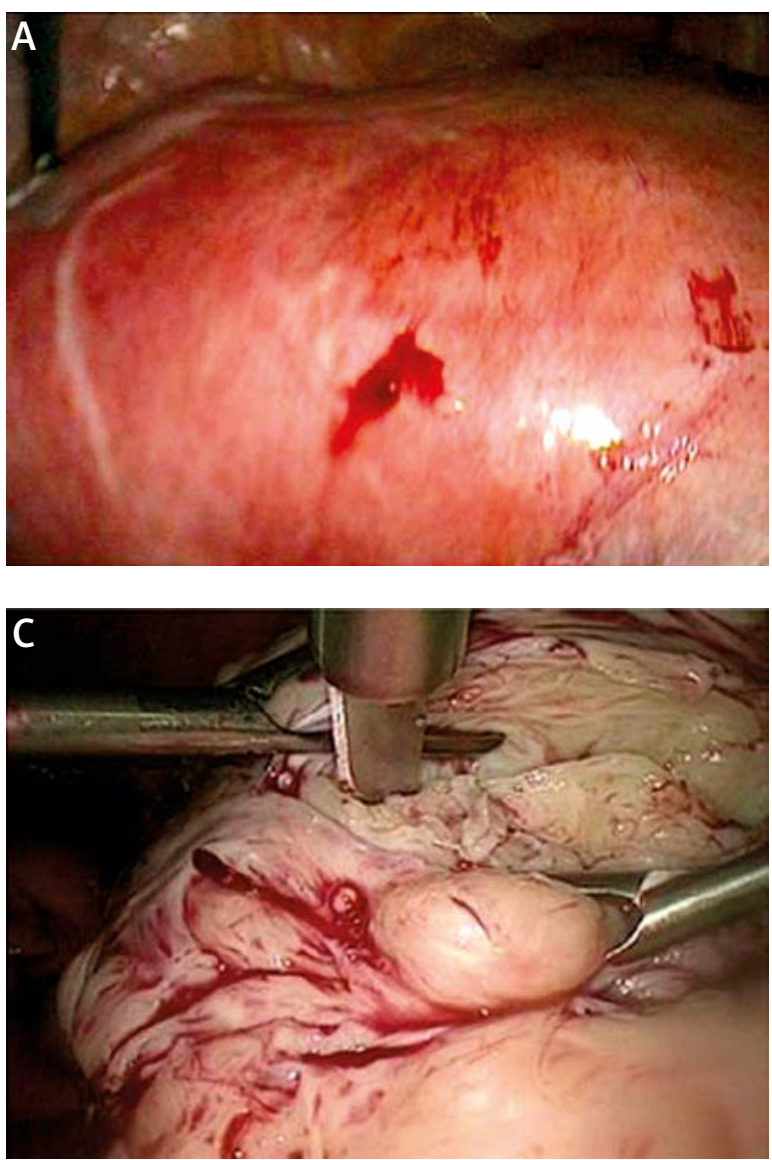

Photo 1. A - Laparoscopic picture of the huge uterus taken from the right upper quadrant. B - Utero-omental adhesions. C - Laparoscopic blade - morcellation. D - Uterine fragments weighed after formalin fixation. The weight of the fixed specimen is lower than the weight of $3030 \mathrm{~g}$, detected postoperatively for the fresh specimen
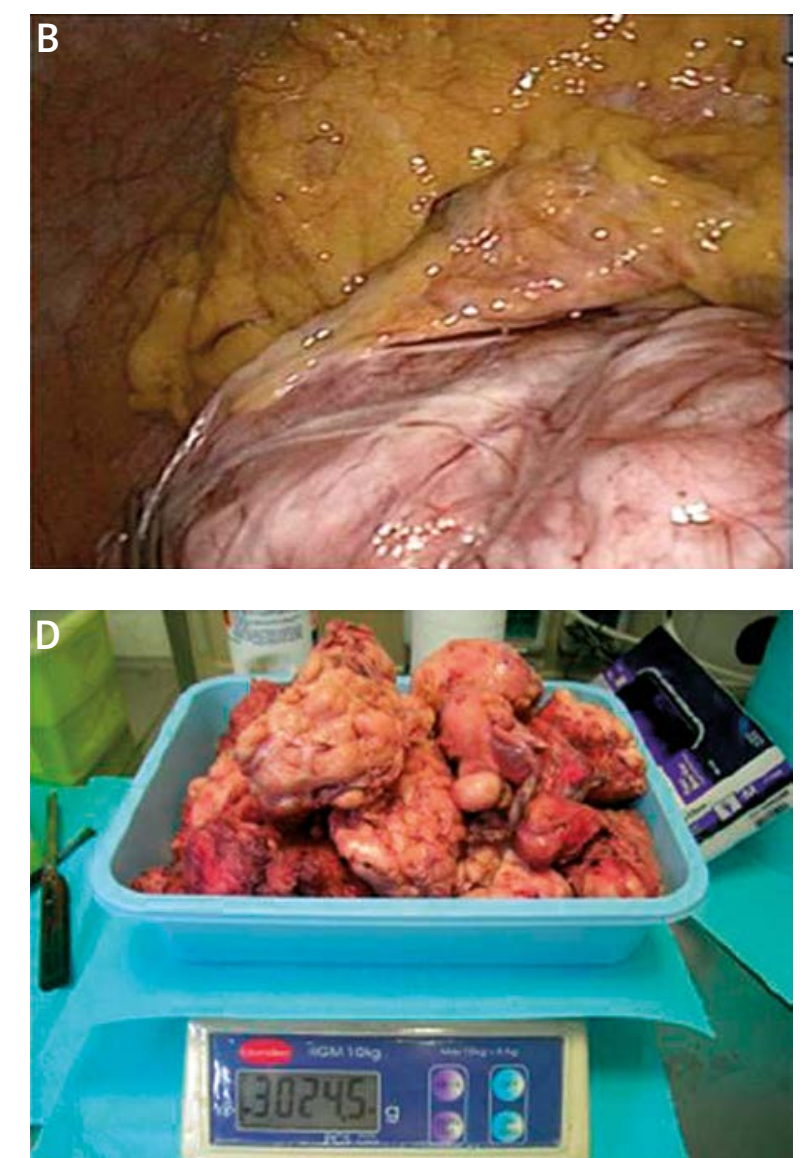

was retrieved in the cul-de-sac. The uterus presented thick adhesions with the bladder, the rectosigmoid, the caecum and the omentum (Photo 1 B), which were cauterized and transected.

The tubes, the utero-ovarian, utero-sacral and round ligaments were identified and coagulated in order to reduce the blood flow to the uterus. Afterward, the utero-ovarian and round ligaments were transected bilaterally and, after opening the posterior leaf of the broad ligament, the utero-sacral ligaments were transected to mobilize the uterus. The anterior leaves of the broad ligament were opened and the vesico-uterine fold was developed. The uterine vessels were then cauterized and transected just medially to the course of the ureters. Finally, cauterization and transection of the cardinal ligaments with paracervical vessels were performed. The cervix at its limit with the vaginal vault was incised, completing 
the extrafascial total laparoscopic hysterectomy. This phase of the operation took approximately $90 \mathrm{~min}$.

As no myoma appeared suspicious for malignancy, laparoscopic cold-knife morcellation of the uterus was performed (Photo $1 \mathrm{C}$ ); however, the extreme resistance of the tissue to morcellation led us to use a laparoscopic cold-blade scalpel to slice the uterus into 16 parts of about $8 \mathrm{~cm}$ each. Because of stenosis of the vagina, vaginal extraction of the uterine fragments was not possible. Furthermore, laparoscopic extraction using a $15-\mathrm{mm}$ morcellator was not possible, due to the hardness of the myometrial tissue. Therefore, an ultra-low mini-laparotomic access of $4 \mathrm{~cm}$ was performed immediately above the pubic bone and all the 16 parts were then manually extracted under laparoscopic vision. Colporraphy was completed laparoscopically by two semi-continuous adsorbable sutures. Vesical and ureteral integrity tests (filling the bladder with $240 \mathrm{ml}$ of methylene blue and with endovenous indigo carmine injection, respectively) were negative, as was the rectal integrity test (bubble test). A cystoscopy was finally performed, finding a double right ureteral meatus but normal ureteral ejaculation and normal bladder mucosa. An intraperitoneal drainage was placed, which was removed on postoperative day 2. Total blood loss was less than $100 \mathrm{ml}$ and the whole surgical time was $300 \mathrm{~min}$. The dry weight of the uterus was $3030 \mathrm{~g}$ (Photo 1 D). The patient had an uneventful postoperative stay and was discharged from hospital three days after surgery.

Haemoglobin level on that day of discharge was $10.8 \mathrm{~g} / \mathrm{dl}$. At 1-month follow-up the patient reported no complications after discharge and a prompt recovery.

\section{Discussion}

The first total laparoscopic hysterectomy (TLH) was performed by Reich et al. in 1989. Since then, several authors have reported their experience with this operation [3-6], thus demonstrating that it can be reproduced.

In a report, Wattiez et al. [7] concluded that "the rationale for TLH is to convert abdominal hysterectomy into a laparoscopic procedure and thereby reduce trauma and morbidity". Nowadays, TLH is currently accepted as a feasible and safe way for the treatment of benign uterine pathology as an acceptable alternative to standard abdominal hysterectomy
$[8,9]$. However, most studies set arbitrarily as a limit of uterine size the equivalent of 15-16 weeks of gestation to make a uterus suitable for laparoscopic surgery. This seems to exclude very enlarged uteri from laparoscopic surgery, thus suggesting a laparotomic approach. The reasons for this lie in putative technical difficulties to perform the hysterectomy laparoscopically (as the uterine volume increases, the access to uterine vascular pedicles worsens and increases the risk of complications such as haemorrhage). Other concerns are linked to the risk of accidental bowel and urinary damage due to poor exposure, difficulty in extraction of the uterus, and duration of the procedure [7].

Only two reports on laparoscopic hysterectomy in cases of very large uteri have been published [10,11]. Wattiez et al. [7] reported that TLH can be successfully carried out in most women with very enlarged uteri and within the range of 500-1230 g, uterine size should no longer be considered an absolute contraindication for TLH [7]. They analysed a series of TLH performed in women with very enlarged uteri. Thirty-four consecutive women with uteri weighting $>500 \mathrm{~g}$ were compared with 68 women with uteri weighting $\leq 300 \mathrm{~g}$. No difference was seen in complication rates between groups; operative time was significantly longer $(p<0.001)$ in women with very enlarged uteri than in those with smaller uteri; however, the groups did not differ in day 1 haemoglobin level drop or hospital stay; nor was conversion to laparotomy required in either group. Indeed certain surgical steps must be undertaken to ensure optimal exposure of the operative field and more effective and safer excision of the uterine vascular pedicle.

The only review assessing the feasibility of TLH in case of very large uteri weighting more than $500 \mathrm{~g}$ was published in 2007 by Fiaccavento et al. [12] on 684 performed TLH procedures [12]. The authors compared 149 patients with a uterus weighting less than $350 \mathrm{~g}$ with 100 patients with a uterus weighting more than $500 \mathrm{~g}$. There was a statistically significant increase in terms of operative time and postoperative hospital stay length in the second group without differences in time to recovery between groups. There were no differences in terms of major complications, demonstrating a low rate of complications and urinary tract injuries, thus showing that laparoscopy should be considered when there is a contraindication to the vaginal approach, and also in the case of large uteri. 
Wattiez et al. [7] first introduced several modifications to the current technique for TLH for huge uteri. The key modifications consisted in higher insertion of the optic cannula with consideration of open abdominal entry to minimize the risk of lacerating the uterus. However, this makes it comfortable for the surgeon to operate. The optic cannula should be placed at least $8-10 \mathrm{~cm}$ above the umbilicus and the 30-degree optic can ensure better visualization of uterine pedicles. The middle cannula should be placed through the umbilicus or even higher, while the other two lateral ones should be placed according to uterine size and location of myomas. The uterine manipulator has a pivotal role in cases of very large uteri to optimize the exposure of structures (vessels and ligaments) and can significantly reduce the operating time. In fact, the main challenge is securing uterine vessels and this can be achieved through polar instruments as described by Wattiez et al. [7] and Fiaccavento et al. [12]. In selected cases, ligation or suturing may reduce the blood supply to the uterus before performing bipolar coagulation, reducing the risk of haemorrhage and ureteral injury.

However, these procedures are for selected cases only. Transection of the vaginal cuff can be performed vaginally or laparoscopically; however, better visualization of pelvic anatomic structures is obtained by laparoscopy, resulting in a safer procedure. Key pitfalls of this advanced surgical procedure are as follows.

\section{Nulliparous patient}

Despite all the advantages of vaginal surgery over the abdominal approach, about $70 \%$ of hysterectomies for benign pathologies are carried out laparotomically [13]. Nulliparous patients seldom present the optimal conditions for a vaginal approach for surgeons with average training due to less loose vaginal tissues. Chapron et al. [14] demonstrated that TLH can represent an optimal way to avoid laparotomy when the conditions are against the vaginal approach as in cases of voluminous uteri or those out of proportion to the vagina. In such cases it is necessary to turn to ancillary techniques to reduce the uterine volume such as morcellation, hemisection, enucleation of myomas, or intramyometrial coring. Therefore, it has been suggested to approach large uteri in nulliparous patients through an abdominal route, namely laparotomy.

\section{Obesity}

A relative contraindication to laparoscopy is obesity, because it presents a major difficulty in establishing pneumoperitoneum and anaesthesiological difficulties with the Trendelenburg position in these women. The majority of authors define obesity as a BMI $>30 \mathrm{~kg} / \mathrm{m}^{2}$ and the Gynaecologic Oncology Group (GOG) limits laparoscopic surgery to women with $\mathrm{BMI}<35 \mathrm{~kg} / \mathrm{m}^{2}$. Indeed, it has been reported that in these cases there is an increased chance of conversion to laparotomy whereas the morbidity and hospital stay were similar between lean and obese patients [15].

\section{Mini-laparotomy}

Benedetti-Panici et al. [16] reported that it is possible to improve the quality of the abdominal scar and to reduce hospital stay after abdominal hysterectomy by making some modifications to surgical and anaesthesiological techniques. A low incision just above the pubic hair line and the use of subcuticular suture were reported to be very efficient in masking the abdominal scar. Pre-emptive analgesia, a small incision length and omission of self-retaining retractor contributed to reduced postoperative stay by decreasing nociceptive stimuli and abdominal trauma. A mini-laparotomic approach was found to be feasible in $80 \%$ of women undergoing hysterectomy for benign conditions and conversion to a larger incision occurred in $2 \%$ of eligible patients only. These data support the wide applicability of the procedure once this technique is standardized and adequate preoperative selection of patients is applied.

In this case the myomas could neither be morcellated due to the extreme hardness of the tissue nor extracted through the vagina due to stenosis, making mandatory an ultralow $4 \mathrm{~cm}$ mini-laparotomic access to extract the uterine slices.

\section{Experience of the surgeon}

The Finnish register detailing morbidity of 10,110 hysterectomies categorized by surgical approach reported a significant decrease of complication rates as surgeons' expertise improved [17]. In other reports, the complication rate for experienced laparoscopists in laparoscopic hysterectomy for benign uterine pathology ranged between $5.8 \%$ and $20 \%$ $[18,19]$. However, recent series reported a total com- 
plication rate of 1.3\% [20] and 5.5\% [21]. In these reports, a laparoscopist was considered "experienced" when he or she had carried out at least $30 \mathrm{TLH}$. In our case, the operator (M.C.) had performed more than $150 \mathrm{TLH}$ at the time of this operation.

\section{Very enlarged uterus}

There is only one study that reports a case of total laparoscopic hysterectomy on a very enlarged uterus weighting more than $3000 \mathrm{~g}$ [22]. The procedure was performed in the US. In this case a 47-year-old patient underwent TLH and bilateral adnexectomy for symptomatic fibromatous uterus causing recurrent bleeding. The operation lasted $5 \mathrm{~h} 57 \mathrm{~min}$ and the estimated blood loss was $800 \mathrm{cc}$.

The specimen - "uterus-cervix, right and left ovaries and fallopian tubes" - consisted of more than 200 fragments with a total weight of $3043 \mathrm{~g}$. The patient, discharged the following day, was readmitted on post-operative day 4 due to a subocclusive status and recovered in 3 days after medical treatment.

In our case, the estimated blood loss was $<100 \mathrm{ml}$ and the patient had no post-operative complications and made a satisfactory prompt recovery. As an ovary of an adult woman is reported to weigh between $6 \mathrm{~g}$ and $8 \mathrm{~g}$, our case may represent the largest uterus in Europe removed by total laparoscopic hysterectomy [23].

\section{Guinness record}

A search on the Web (PubMed, ENBASE, Google) was carried out with the words "Guinness uterus". The largest uterus that was reported to be removed laparoscopically was described by Dr Rakesh Sinha of the BEAMS Hospital who broke the earlier Guinness record held by Demir and Marchand, two US doctors who removed a 3.2-kg uterus. The case was reported in the Guinness World Records as a uterus weighting $4.1 \mathrm{~kg}$ that was removed using minimally invasive surgery.

Sinha had a previous personal record in 2005 when he removed a 3.4-kg fibroid. The second surgery was performed in 2009 and Sinha received the Guinness certification on October 21, 2009 [24].

However, in Kochi (Kerala, India), on October 7, 2009, a team of doctors claimed to have removed the largest uterus, weighting $4.9 \mathrm{~kg}$, in a 46 -yearold woman who was suffering from a huge fibroid [25]. The five doctors from the Sunrise Institute of
Medical Sciences (SIMS), with the main surgeon $\mathrm{Ha}$ feez Rahman, achieved the feat using less invasive laparoscopic key hole surgery. A $5 \mathrm{~mm}$ incision was performed on the abdomen and a "morcellator" was used. The doctors reported the details of surgery for entry into the Limca and Guinness Books of World Records. The uterus was removed in only $28 \mathrm{~min}$ with minimal blood loss. The patient was discharged within $24 \mathrm{~h}$ and resumed her work at the bank. The doctors added that the uterus was preserved in the pathological lab of the hospital for professional references. Nevertheless, many details seem contradictory for surgeons who usually perform total laparoscopic hysterectomies. No scientific report exists on PubMed about this case and nor any reference about the time required to laparoscopically remove the specimen.

\section{Suspect of sarcoma}

One of the major concerns in evaluating adequate treatment of rapid growth or symptomatic leiomyomas is the possibility for a uterine sarcoma in histopathology. There is just one study addressing this specific issue, published by Parker et al. [26] on 1332 women who underwent hysterectomy or myomectomy for uterine myomas. This study had a clear bias as the definition of "rapid growth" was unclear. However, the total incidence of uterine sarcoma (leiomyosarcoma, endometrial stromal sarcoma and mixed mesodermal tumour) among 1332 patients operated on for uterine leiomyoma was extremely low $(0.23 \%)$ while the incidence among those with pre-surgical diagnosis of rapidly growing leiomyoma was $0.27 \%$, making no substantial differences as for increased risk for sarcoma when rapid growth is reported. However, in the case of suspicious myomas growing after menopause, growing on $\mathrm{GnRH}$ agonists, rapidly growing over a short time frame in premenopause, exceeding $10 \mathrm{~cm}$ in diameter, caution should be used, maybe evaluating the option of a laparotomic approach to avoid morcellation of the uterus/myomas.

\section{Uterine size in abdominal hysterectomy (AH)}

Uterine size has been preferably assessed by the weight and not by subjective evaluation as the equivalent of pregnant uteri (weeks of gestation) [27]. Hillis et al. [27] reported the complication rate of abdominal hysterectomy for leiomyomas as high 
as a two- to threefold increase in the adjusted odds for blood loss and vaginal cuff cellulitis between the two groups, namely uteri weighting more (57\%) and less $(43 \%)$ than $500 \mathrm{~g}$. Indeed, these percentages are far higher than those reported in more recent series as underlined by Carlson in 1994 [28]. Furthermore, it has to be highlighted that the uterine size also affects the choice of kind of abdominal incision (longitudinal versus transverse). Thus, considering our case, the only abdominal approach for performing hysterectomy should have been through a supra-umbilical or xifo-pubic longitudinal incision.

\section{Conclusions}

Total laparoscopic hysterectomy can be performed even in very large uteri when the simultaneous presence of an expert surgeon and appropriate instruments can be assured, with no increase in complication rates. Furthermore, a prompt recovery that is similar to that of women with moderately enlarged uteri is reported. Although there is an increase in duration of surgery in cases of very enlarged uteri, the lower post-operative complication rates compared to the classical laparotomic approach suggest the possibility of laparoscopic treatment whenever the conditional requirements (surgeon and instruments) are met.

\section{References}

1. Carlson KJ, Nichols DH, Schiff I. Indications for hysterectomy. N Engl Med J 1993; 328: 856-60.

2. Carter JE, McCarus SD. Laparoscopic myomectomy. Time and cost analysis of power vs. manual morcellation. J Reprod Med 1997; 42: 383-8.

3. Summitt RL, Stovall TG, Lipscomb GH, Ling FW. Randomized comparison of laparoscopy-assisted vaginal hysterectomy with standard vaginal hysterectomy in an outpatient setting. Obstet Gynecol 1992; 80: 895-901.

4. Daniell JF, Kurtz BR, McTavish G, et al. Laparoscopically assisted vaginal hysterectomy. The initial Nashville, Tennessee, experience. J Reprod Med 1993; 38: 537-42.

5. Reich H, Mc Glynn F, Sekel L. Total laparoscopic hysterectomy. Gynaecol Endosc 1993; 2: 59-63.

6. Chapron C, Dubuisson JB, Aubert V, et al. Total laparoscopic hysterectomy preliminary results. Hum Reprod 1994; 9: 2084-9.

7. Wattiez A, Soriano D, Fiaccamento A, et al. Total laparoscopic hysterectomy for very enlarged uteri. J Am Assoc Gynecol Laparosc 2002; 9: 125-30.

8. Olsson JH, Ellstrom M, Hahlin M. A randomized prospective trial comparing laparoscopic and abdominal hysterectomy. $\mathrm{Br}$ J Obstet Gynaecol 1996; 103: 345-50.

9. Seracchioli R, Venturoli S, Vianello F, et al. Total laparoscopic hysterectomy compared with abdominal hysterectomy in the presence of a large uterus. J Am Assoc Gynecol Laparosc 2002; 9: 333-8.

10. Pelosi MA, Kadar N. Laparoscopically assisted hysterectomy for uteri weighting 500g or more. J Am Assoc Laparosc 1994; 1: 405-9.

11. Salmanli N, Maher P. Laparoscopically-assisted vaginal hysterectomy for fibroid uteri weighing at least 500 grammes. Aust N Z J Obstet Gynaecol 1999; 39: 182-4.

12. Fiaccavento A, Landi S, Barbieri F, et al. Total laparoscopic hysterectomy in cases of very large uteri: a retrospective comparative study. J Minim Inv Gynecol 2007; 14: 559-63.

13. Dicker RC, Scally MJ, Greenspan JR, et al. Hysterectomy among women of reproductive age. Trends in the United States, 19701978. J Am Med Assoc 1982; 248: 323-7.

14. Chapron C, Dubuisson JB, Ansquer Y. Hysterectomy for patients without previous vaginal delivery: results and modalities of laparoscopic surgery. Hum Reproduct 1996; 11: 2122-6.

15. Eltabbakh GH, Piver MS, Hempling RE, Recio FO. Laparoscopic surgery in obese women. Obstet Gynecol 1999; 94: 704-8.

16. Benedetti-Panici PL, Zullo MA, Angioli R, Muzii L. Minilaparotomy hysterectomy: a valid option for the treatment of benign uterine pathologies. Obstet Gynecol 2005; 119: 228-31.

17. Mäkinen J, Johansson J, Tomás C, et al. Morbidity of 10110 hysterectomies by type of approach. Hum Reprod 2001; 16: 1473-8.

18. Jansen FW, Kapiteyn K, Trimbos-Kemper T, et al. Complications of laparoscopy: a prospective multicentre observational study. Br J Obstet Gynaecol 1997; 104: 596-600.

19. Chapron CM, Dubuisson JB, Ansquer Y. Is total laparoscopic hysterectomy a safe surgical procedure? Hum Reprod 1996; 11: 2422-4.

20. Wattiez A, Soriano D, Cohen SB, et al. The learning curve of total laparoscopic hysterectomy: comparative analysis of 1647 cases. J Am Assoc Gynecol Laparosc 2002; 9: 339-45.

21. Malzoni M, Perniola G, Perniola F, Imperato F. Optimizing the total laparoscopic hysterectomy procedure for benign uterine pathology. J Am Assoc Gynecol Laparosc 2004; 11: 211-8.

22. Walid MS, Heaton RL. Laparoscopic extirpation of a 3-Kg uterus. Arch Gynecol Obstet 2009; 279: 607-8.

23. Bairati A. Trattato di Anatomia Umana. Morfologia Generale e sistematica dell'uomo. Vol. VI, II Ed. Minerva Medica.

24. Available at: http://timesofindia.indiatimes.com/city/mumbai/ Doc-removes-41-kg-uterus28makes-it-to-Guinness-records-/ articleshow/5194317.cms.

25. Available at: http://www.worldamazingrecords.com/2009/10/ 49-kg-worlds-largest-uterus-set30world.html.

26. Parker WH, Fu YS, Berek JS. Uterine sarcoma in patients operated on for presumed leiomyoma and rapidly growing leiomyoma. Obstet Gynecol 1994; 83: 414-8.

27. Hillis SD, Marchbanks PA, Peterson HB. Uterine size and risk of complications among women undergoing abdominal hysterectomy for leiomyomas. Obstet Gynecol 1996; 87: 539-43.

28. Carlson KJ, Miller BA, Fowler FJ. The 1 Maine women's health study I: outcomes of hysterectomy. Obstet Gynecol 1994; 83: 5-65.

Received: 10.04.2013, accepted: 21.12.2013. 